\title{
4D flow MRI can detect subtle right ventricular dysfunction in primary left ventricular disease.
}

\author{
Alexandru Grigorescu Fredriksson, Emil Svalbring, Jonatan Eriksson, Petter Dyverfeldt, \\ Urban Alehagen, Jan Engvall, Tino Ebbers and Carl-Johan Carlhäll
}

\section{Linköping University Post Print}

\section{Tweet}

N.B.: When citing this work, cite the original article.

Original Publication:

Alexandru Grigorescu Fredriksson, Emil Svalbring, Jonatan Eriksson, Petter Dyverfeldt, Urban Alehagen, Jan Engvall, Tino Ebbers and Carl-Johan Carlhäll, 4D flow MRI can detect subtle right ventricular dysfunction in primary left ventricular disease., 2015, Journal of Magnetic Resonance Imaging.

http://dx.doi.org/10.1002/jmri.25015

Copyright: Wiley

http://eu.wiley.com/WileyCDA/

Postprint available at: Linköping University Electronic Press

http://urn.kb.se/resolve?urn=urn:nbn:se:liu:diva-124293 


\section{D flow MRI can detect subtle right ventricular dysfunction in primary left ventricular disease}

Alexandru G. Fredriksson MD ${ }^{1,2 *}$; Emil Svalbring ${ }^{1}$; Jonatan Eriksson $\mathrm{PhD}^{1,3}$; Petter Dyverfeldt $^{1,3} \mathrm{PhD}$; Urban Alehagen ${ }^{1,4} \mathrm{MD}$, PhD; Jan Engvall ${ }^{1,3,5} \mathrm{MD}$, PhD; Tino Ebbers $^{1,3,6} \mathrm{PhD}$; Carl-Johan Carlhäll MD, PhD $\mathrm{D}^{1,3,5}$

${ }^{1}$ Division of Cardiovascular Medicine, Department of Medical and Health Sciences, Linköping University, Linköping, Sweden.

${ }^{2}$ Research and Development Unit, Örebro University Hospital, Örebro, Sweden.

${ }^{3}$ Center for Medical Image Science and Visualization (CMIV), Linköping University, Linköping, Sweden.

${ }^{4}$ Department of Cardiology, Department of Medical and Health Sciences, Linköping University, Linköping, Sweden.

${ }^{5}$ Department of Clinical Physiology, Department of Medical and Health Sciences, Linköping University, Linköping, Sweden.

${ }^{6}$ Division of Media and Information Technology, Department of Science and Technology, Linköping University, Linköping, Sweden.

${ }^{*}$ Corresponding author

Alexandru Fredriksson, MD

Division of Cardiovascular Medicine

Department of Medical and Health Sciences

Linköping University

SE - 58183 Linköping

E-mail: alexandru.fredriksson@liu.se 
Grant support: the Swedish Heart and Lung foundation, the Swedish Research Council, the European Union FP7 project number 223615, the Medical Research Council of Southeast Sweden (FORSS), the County Council of Ostergotland/Heart and Medicine Center.

Running title: 4D flow MRI can detect subtle RV dysfunction in primary LV disease. 


\section{Abstract}

\section{Purpose}

To investigate whether 4D flow MRI can detect subtle right ventricular (RV) dysfunction in primary left ventricular (LV) disease.

\section{Methods}

4D flow and morphological 3T MRI data were acquired in 22 patients with mild ischemic heart disease, that were stratified into two groups based on LV end-diastolic volume index (EDVI): lower-LVEDVI and higher-LVEDVI, as well as in 11 healthy controls. The RV volume was segmented at end-diastole (ED) and end-systole (ES). Pathlines were emitted from the ED volume and traced forwards and backwards in time to ES. The blood volume was separated into flow components. The Direct Flow (DF) component was defined as RV inflow passing directly to outflow. The kinetic energy (KE) of the DF component was calculated. Echocardiographic conventional RV indices were also assessed.

\section{Results}

The higher-LVEDVI group had larger LVEDVI and lower LV ejection fraction (98 $\pm 32 \mathrm{ml} / \mathrm{m}^{2} ; 48 \pm 13 \%$ ) compared to the healthy (67 $\pm 12, \mathrm{P}=0.002 ; 64 \pm 7, \mathrm{P}<0.001$ ) and lower-LVEDI group $(62 \pm 10 ; 68 \pm 7$, both $\mathrm{P}<0.001)$. The RV 4D flow specific measures "DF/EDV volume-ratio" and "DF/EDV KE-ratio at ED" were lower in the higher-LVEDVI group ( $38 \pm 5 \% ; 52 \pm 6 \%$ ) compared to the healthy (44 $\pm 6 ; 65 \pm 7$, $\mathrm{P}=0.018$ and $\mathrm{P}<0.001)$ and lower-LVEDVI groups $(44 \pm 6 ; 64 \pm 7, \mathrm{P}=0.011$ and $\mathrm{P}<0.001)$. There was no difference in any of the conventional MRI and echocardiographic RV indices between the three groups. 


\section{Conclusion}

We found that in primary LV disease, mild impairment of RV function can be detected by $4 \mathrm{D}$ flow specific measures, but not by the conventional MRI and echocardiographic indices.

Keywords: 4D flow MRI, right ventricular function, interventricular interaction, left ventricular disease 


\section{INTRODUCTION}

Right ventricular (RV) function has a significant prognostic value in both acquired and congenital heart disease (1). The prognostic value of RV function in primary right-sided conditions, such as pulmonary hypertension and cardiomyopathies, is readily acknowledged but RV dysfunction has also been linked to higher mortality and morbidity in patients with primary left ventricular (LV) disease. Multiple studies have shown that RV function is a predictor for survival in many primary LV disease states, such as acute coronary disease, ischemic cardiomyopathy and congestive heart failure (2-6). Poor RV function may further aggravate LV dysfunction, consequently leading to disease progression (7).

Echocardiography is still the most available and thus the first line of cardiac imaging modalities in the assessment of RV function. However, echocardiographic assessment of $\mathrm{RV}$ function is challenging due to the retrosternal location and complex crescentshaped geometry of the RV (8-10). Although the primary purpose of the heart is to generate blood flow through the cardiovascular system (11), the assessment of RV function is still mainly based on cardiac morphological data $(8,9)$, and rarely based on blood flow data. Echo Doppler assessment of blood flow is limited to one spatial velocity component over time, resulting in angle dependence, which is cumbersome for the complex RV blood flow.

Three-dimensional (3D) time-resolved phase-contrast MRI with three-dimensional motion encoding permits flow assessment of the true 3D nature of intracardiac blood flow over the cardiac cycle (12-17). A recent 4D (3D + time) flow MRI study of the normal RV described patterns and energetics of RV flow with a special emphasis on 
RV diastolic inflow passing directly to ensuing outflow (Direct Flow). It was suggested that the preservation of end-diastolic kinetic energy (KE) of the RV Direct Flow reflects favorable conditions for an effective systolic ejection (18).

In primary LV disease several mechanisms may contribute to compromised RV function. The afterload of the RV may increase due to elevated LV filling pressures, but RV function may also be hampered due to interventricular interaction, in turn secondary to myocardial dysfunction and pericardial constraint $(19,20)$. Consequently, visualization and quantification of RV 4D flow patterns and energetics in patients with primary left ventricular disease has the potential to add to detection of early impairment of RV function.

Accordingly, we sought to study RV 4D flow patterns and energetics, with a special emphasis on RV inflow passing directly to ensuing outflow (Direct Flow), and hypothesized that such flow specific markers of RV function could detect subtle impairment of RV function in patients with primary LV disease.

\section{METHODS}

\section{Study sample}

A total of twenty-two patients with diagnosed or clinical suspicion of chronic ischemic heart disease based upon pathological stress test results (myocardial perfusion scintigraphy or ECG exercise testing) with typical ischemic appearance and/or a presentation with clinical symptoms of chronic ischemic heart disease such as fatigue, dyspnea, stable angina together with the fulfillment of the high risk score of CVD of the European Society of Cardiology were included in the study. The 
patients were enrolled from outpatients at the Department of Cardiology, Linköping University Hospital. In addition, eleven healthy controls with no history of prior or current cardiovascular disease or cardiac medication and a normal echocardiogram were included (Table 1).

The patient group was for comparative purposes further divided into two equal strata with eleven patients each based on their LV end-diastolic volume index (EDVI) value as a measure of LV remodeling; one group of patients presented lower values (lowerLVEDVI, $\leq 74 \mathrm{ml} / \mathrm{m}^{2}$ ) and one group of patients presented higher values (higherLVEDVI, $\geq 74 \mathrm{ml} / \mathrm{m}^{2}$ ), (Table 2) (21).

Exclusion criteria for all groups were contraindication for MRI-examination, significant ventricular arrhythmia, heart rate $<40$ bpm respectively $>100$ bpm or more than mild right-sided valvular disorder. Exclusion criterion for the patient group was also any acute coronary syndrome (STEMI/Non-STEMI) during the last three months prior to data collection. The study was approved by the regional Ethical Review Board in Linköping, Sweden and all participants gave written informed consent.

\section{Data acquisition}

A 3T MRI scanner (Philips Ingenia; Philips Medical Systems, Best, the Netherlands) was used for the acquisition of 4D flow data and morphological short-axis, apical long-axis as well as two- and four-chamber images. The 4D flow data was acquired during free breathing using a retrospective vector-ECG- and navigator-gated phase contrast sequence. Data acquisition was triggered at end-expiratory phase. Scan parameters were: $\mathrm{VENC}=120 \mathrm{~cm} / \mathrm{s}$, flip angle $=10^{\circ}$, echo time $=2.6 \mathrm{~ms}$, repetition 
time $=4.4 \mathrm{~ms}$, SENSE factor $=3$ and $k$-space segmentation factor $=3$. The spatial resolution was $2.8 \times 2.8 \times 2.8 \mathrm{~mm}^{3}$. The field of view was adjusted to cover the whole heart for each subject. Scan time was approximately 16-20 min including 50\% average navigator efficiency at heart rate 60 bpm, nominal time was approximately 810 min. Concomitant gradient field effects were corrected on the scanner. Phase wraps and background phase offsets were corrected with automated in-house postprocessing software. The background phase offset correction comprised a secondorder weighted least-squares fit to the static tissue, which was previously found to provide the most robust correction for the RV $(18,22)$.

The morphological images were acquired during end-expiratory breath holds in 30 time frames using bSSFP imaging. The short- and long-axis images had an acquired resolution of $1 \times 1 \mathrm{~mm}$ and a slice thickness of $8 \mathrm{~mm}$. The number of slices varied according to the size of each patient's heart.

A conventional transthoracic echocardiographic examination using a Vivid E9 scanner (GE, Vingmed Ultrasound, Horten, Norway) was also performed. For exclusion criteria purposes, parameters of right-sided valve function were acquired. For comparative purposes, parameters of left and right systolic and diastolic ventricular function were acquired. Three consecutive heartbeats were acquired during free breathing. The examinations were performed by experienced ultrasound technicians at the Department of Clinical Physiology, Linköping University Hospital.

\section{Data analysis}


The MRI data was analyzed using a semi-automatic method described by Eriksson et al. (22) and previously evaluated for intra-ventricular 4D flow $(18,22)$. The RV was segmented at the time of end-diastole (ED) and end-systole (ES) using the free

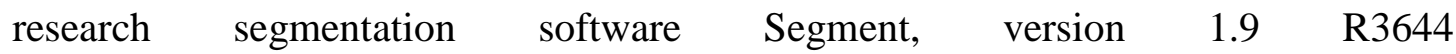
(http://www.medviso.com) (23); timeframes were visually defined in the fourchamber image as the first time frame in which the tricuspid valve was closed after diastolic filling and the last time frame before tricuspid opening after systolic ejection, respectively. The segmentation was performed in the short-axis images with guidance by the long-axis images and followed the contour of the compact myocardium. Two RV volumes, end-diastolic and end-systolic (EDV and ESV) were obtained. The LV was segmented in similar fashion for stratification purposes.

The ESV and EDV were resampled to match the spatial resolution of the 4D velocity data. Pathlines, representing the paths of virtual blood flow particles, were emitted from the center of each voxel inside the EDV and traced forwards and backwards in time to the time of ES, thus encompassing the whole cardiac cycle. The pathlines were calculated with a $4^{\text {th }}$ order Runge-Kutta method with 5 ms step length using inhouse software. The pathlines were considered to represent the whole intraventricular RV blood volume, which was subsequently automatically divided into functional blood flow components by using the ES segmentation and its most basal plane to determine the origin and destination of each pathline (22). In this way the RV blood volume could be divided into four previously described ventricular flow components, based upon the transition route through the ventricle $(12,18)$ :

- Direct Flow, the blood flow volume that enters the ventricle and is ejected during the analyzed heartbeat. 
- Retained Inflow, the blood flow volume that enters, but does not leave the ventricle during the analyzed heart beat.

- Delayed Ejection Flow, blood flow volume that resides within the ventricle at the beginning of and is ejected during the analyzed heart beat.

- Residual Volume, blood flow volume that re-circulates within the ventricle for two or more heart beats.

In this study the main focus will lay on the Direct Flow component. A commercially available flow visualization software (EnSight, CEI Inc, Apex, North Carolina, USA) was used to visualize the RV pathlines over time (24).

The kinetic energy (KE) was calculated for the Direct Flow component over the entire cardiac cycle as the sum of KE for all pathlines included in this component using the volume represented by each pathline, the density of blood and the velocity of the pathline in every time point.

All datasets were subjected to careful quality control and emitted pathlines were visually inspected for artefacts or MRI derived inconsistency. Ventricular inflow and outflow volumes were compared for each subject. An inflow-outflow discrepancy of $>15 \%$, mainly due to subject movement inside the scanner resulting in a positional mismatch between the morphological and 4D flow data, rendered exclusion.

The echocardiographic data were analyzed offline using the manufacturer analysis software (EchoPac, v112, GE Healthcare). All parameters were measured in three heartbeats and the mean value was registered. Early diastolic peak velocities of transmitral inflow (E) and mitral annulus (é) were measured using pulse wave 
Doppler and color-coded tissue Doppler, respectively. The RV basal diameter was defined as the maximal dimension in the most basal third of the chamber at enddiastole. RA area was traced at the ventricular end-systolic timeframe defined as the timeframe before tricuspid valve opening. The tricuspid annular plane systolic excursion (TAPSE) was measured using M-mode in the free wall. Early and late diastolic (é and á) and systolic (s') peak velocities of the tricuspid annulus were measured in the RV free wall using color-coded tissue Doppler.

\section{Statistics}

All values are given as group means \pm 1 SD. For comparison of parameters between the study groups, a one-way ANOVA with Ficher Least Significant Difference posthoc test was used. The statistical significance level was set at $\mathrm{P}<0.05$. The software used for all statistical analysis was Statistica 9.1 (Statsoft Inc., Tulsa, Oklahoma, USA).

\section{RESULTS}

\section{Descriptive parameters}

The age of the subjects was slightly higher in the lower-LVEDVI group compared to the control group ( $\mathrm{P}=0.016)$ (Table 1). The systolic blood pressure was higher in the lower-LVEDVI group compared with the control group and the higher-LVEDVI groups ( $\mathrm{P}=0.012$ and $\mathrm{P}=0.024$ ) (Table 1 ). The diastolic blood pressure was higher in the lower-LVEDVI group compared with the higher-LVEDVI group $(\mathrm{P}=0.011)$. No other statistically significant difference between the study groups was found. Almost all patients were clinically compensated and presented NYHA I-II; only three patients 
presented NYHA class III. The RV inflow and outflow mean values for the whole study sample were $68.4 \pm 17.2 \mathrm{ml}$ and $65.1 \pm 15.5 \mathrm{ml}$, respectively.

\section{Conventional MRI derived parameters and LV Elé}

There was no statistically significant difference between the three groups in RV enddiastolic volume index (RVEDVI) (Healthy vs lower-LVEDVI, $\mathrm{P}=0.207$; Healthy vs higher-LVEDVI, 0.736; lower-LVEDVI vs higher-LVEDVI, $\mathrm{P}=0.113$ ) and RV ejection fraction $(\mathrm{RVEF})(\mathrm{P}=0.498 ; \mathrm{P}=0.077 ; \mathrm{P}=0.260)$ derived from MRI (Figure 1, Table 2). However, LVEDVI was significantly higher and LVEF were significantly lower in the higher-LVEDVI group compared to the lower-LVEDVI group (both $\mathrm{P}<0.001)$ and the healthy control group $(\mathrm{P}=0.002$ and $\mathrm{P}<0.001)$. The LV E/é-ratio, derived from the echocardiographic examination as a description of the LV diastolic function, was significantly higher in the lower-LVEDVI and higher-LVEDVI groups compared to the healthy control group $(\mathrm{P}=0.005$ and $\mathrm{P}=0.018$, respectively). No difference was found in this parameter between the lower-LVEDVI and higherLVEDVI groups.

\section{RV echocardiographic parameters}

There was no significant difference in any of the RV echocardiographic parameters reflecting systolic or diastolic function between the three groups: RV basal diameter (Healthy vs lower-LVEDVI, $\mathrm{P}=0.352$; Healthy vs higher-LVEDVI, $\mathrm{P}=0.143$; lowerLVEDVI vs higher-LVEDVI, $\mathrm{P}=0.565)$, é/á ratio ( $\mathrm{P}=0.137 ; \mathrm{P}=0.081 ; \mathrm{P}=0.720)$, RA area $(\mathrm{P}=0.545 ; \mathrm{P}=0.355 ; 0.722), \mathrm{RV}$ fractional area change $(\mathrm{P}=0.792 ; \mathrm{P}=0.773$; $\mathrm{P}=0.596)$, TAPSE ( $\mathrm{P}=0.708 ; \mathrm{P}=0.526 ; \mathrm{P}=0.787), \mathrm{RV} \mathrm{s}^{\prime}(\mathrm{P}=0.356 ; \mathrm{P}=0.206 ; \mathrm{P}=0.690)$ (Table 3). 


\section{Visual description of RV flow}

In the healthy group the visualization of RV flow component distribution throughout diastole presented an organized pattern. As previously observed (18), an asymmetric ring vortex surrounds the tricuspid inlet during early and late diastolic filling. During early filling, a substantial portion of the Direct flow (green) component rounds the infundibular septum constituting the superior portion of the ring vortex, which persists through the diastasis and gains speed at late filling, propelling the Direct flow towards the pulmonary valve (Figure 2, Supplemental movie 1). The Direct flow component was at ED mainly situated centrally in the basal half of the chamber extending towards the RVOT. The Retained inflow (yellow) pursued a more inferiolateral path in the chamber. The Delayed ejection flow component (blue) was situated in the superior part of the ventricle at onset diastole and was further pushed towards the pulmonary valve with the inflowing Direct flow. The Residual volume (red) resided in the apical and peripheral regions of the ventricle and appeared to be pushed apically by the inflowing Retained inflow.

In the higher-LVEDVI group the flow component distribution appeared to be less organized with higher degrees of mixing between components. This pattern was most evident in the patients with the highest LVEDVI values $\left(>100 \mathrm{ml} / \mathrm{m}^{2}\right)$. Moreover, in these patients at late diastole the inflowing blood in the superior portion of the tricuspid ring vortex was divided into a major counter clockwise flow path up into the RVOT (mainly Direct flow) but also into a minor clockwise flow path down towards the apical part of the interventricular septum (viewed from the lateral aspect of the RV) (Figure 3, Supplemental movie 2). 


\section{RV 4D flow specific parameters}

The Direct Flow as percentage of total EDV (DF/EDV volume-ratio), was significantly lower in the higher-LVEDVI group compared to the healthy control group ( $\mathrm{P}=0.018)$ and the lower-LVEDVI group $(\mathrm{P}=0.011)$ (Figure 1, Table 4). The Retained Inflow as percentage of total EDV was significantly higher in the higherLVEDVI compared to the lower-LVEDVI group $(\mathrm{P}=0.05)$ and this parameter tended to be higher in the higher-LVEDVI group compared to the control group. The Delayed Ejection Flow as percentage of total EDV was significantly higher in the higher-LVEDVI group and the lower-LVEDVI group compared to the healthy control group ( $\mathrm{P}=0.009$ and $\mathrm{P}=0.031$, respectively). There was no intergroup difference in the Residual Volume as percentage of total EDV. The KE possessed by the Direct Flow as percentage of the KE possessed by total EDV (DF/EDV KE-ratio) at ED was significantly lower in the higher-LVEDVI group compared to the healthy control group $(\mathrm{P}<0.001)$ and the lower-LVEDVI group $(\mathrm{P}<0.001)$ (Figure 1 , Table 4$)$.

\section{DISCUSSION}

In the present study RV 4D flow patterns and energetics were assessed in patients with primary left-sided chronic ischemic heart disease. The findings propose that RV 4D flow specific measures allow for detection of subtle impairment of RV function in patients with primary LV disease.

The healthy controls in this study at 3.0 Tesla presented values of the relative amount of Direct flow (DF/EDV volume ratio) that was similar to previously published values in healthy controls at 1.5 Tesla (18). Overall, the patients in the present study were 
well managed and showed only a mild severity of disease with respect to functional classification (NYHA) and LV configuration and function, creating possibilities for detection of subtle alterations of RV function. In the higher-LVEDVI group a lower relative amount of RV Direct Flow was detectable, suggesting that the RV hemodynamics are altered in these patients compared to the healthy subjects and the lower-LVEDVI group. This lower relative amount of Direct flow is due to RV inflow being retained in the ventricular cavity instead of passing directly to the RVOT and ejection. As previously introduced there may be several reasonable mechanisms underlying the RV hemodynamic changes seen in these patients with primary LV disease.

It is well known that the LV plays a pivotal role in systolic RV flow and pressure generation and that the most common cause of RV failure is primary LV dysfunction (1). Failure of the LV due to ischemic myocardial injury may eventually lead to diastolic ventricular dysfunction, elevated LV filling pressures and congestion of the pulmonary circulatory system, thus in turn pressure overloading the RV (19). However, it is not reasonable to believe that the main explanation for the hemodynamic alteration in the higher-LVEDVI group seen in the present study is merely due to increased RV afterload secondary to increased LV filling pressures and pulmonary hypertension. Both patient groups had higher LV E/é values than the control group, but the measured values were still below the level where significant elevated filling pressures and pulmonary congestion are likely to be present (25). Moreover, no difference was found between the groups regarding the RVEF, which has been found to be inversely correlated to RV afterload (2). 
The subepicardial oblique muscle fibers in the LV contribute to augmented RV function mediated through the shared interventricular septum (7). Ischemic LV disease may eventually result in myocardial remodeling, morphologically seen as chamber dilatation (26) which can lead to increased transversing of the oblique muscle fibers in the interventricular septum $(19,27,28)$. Hence, the observed RV intraventricular flow alteration could in part be explained by altered properties of the shared interventricular septum in the patients with higher LVEDVI. Of note, in the patients with the highest degree of $\mathrm{LV}$ remodeling $\left(>100 \mathrm{ml} / \mathrm{m}^{2}\right)$, the late inflowing blood in the superior portion of the tricuspid ring vortex divided into a major counter clockwise flow path up into the RVOT (mainly Direct flow) but also into a minor clockwise flow path. This later "abnormal” clockwise flow path was located in the vicinity of the interventricular septum and it could be speculated that altered properties of the shared interventricular septum may redirect a portion of the late inflowing blood away from the RVOT, and thus reducing the amount of Direct Flow.

The relative amount of KE possessed by the Direct flow component (DF/EDV KE ratio) at ED was lower in the higher-LVEDVI group compared to the other two groups. Although the absolute values of the KE possessed by the Direct flow are much lower than the estimated stroke work of the RV (29), a preservation of KE at ED reflects a transit of inflowing blood though the RV that may be favorable for an efficient systolic ejection (18). Hence, a lower relative amount of KE possessed by the Direct flow at ED in the higher-LVEDVI patients, suggests that a lower proportion of the RV EDV contributes to efficient systolic ejection. Moreover, this suggests that a larger relative amount of the $\mathrm{KE}$ of the $\mathrm{RV}$ inflowing blood in these patients is transferred into other flow components or other energy forms. This fact may reflect a 
less efficient RV diastolic-systolic coupling in a heart with a remodeled LV and compromised septal function (30).

There was no statistical significant difference in any of the echocardiographic parameters reflecting systolic or diastolic function between the three groups. As many conventional echocardiographic parameters for assessment of RV function are based on RV free wall measurements, such as TAPSE, s', é and á $(8,9)$, a compromised septal function due to interventricular interaction may be overseen, and thus, global RV function may not be fully assessed. An advantage of RV 4D flow measurements, and in particular the Direct Flow component, is that not only the RV free wall and interventricular septum performance is taken into account, but the configuration and function of the entire ventricular chamber and its interaction with flow.

The present findings relate only to a relatively small number of patients and healthy subjects in the supine position at rest and in sinus rhythm with no significant valvular disorder. All MRI data were acquired during end-expiration and results may vary over the respiratory cycle. Echocardiographic data were acquired during free breathing, which especially may affect the RV diastolic function parameters sensitive to respiration, such as RA area and E values. All echocardiographic values were averaged over three consecutive heartbeats to reduce some of these variations. Some patients with cardiac disease may have difficulties to fully comply during the MRI examinations, thus giving rise to higher levels of motion artefacts or data mismatch. Acquisition of double short-axis image stacks, one before and one after the flow acquisition, was carried out to minimize the risk of mismatch. A slightly higher acceptance window for inflow-outflow discrepancy was allowed in this study, 
although with rigorous quality control and robust results. Careful quality control included observation for e.g. aberrant pathlines and significant differences between inflow- and outflow-volumes. The study groups were not completely gender and age matched. There were fewer women in the higher-LVEDVI group than in the healthy control and the lower-LVEDVI groups. The healthy control group was younger than the lower-LVEDVI group. However the absolute ages of the participants were fairly well matched.

In conclusion, this pilot study shows that in primary LV disease, subtle impairment of RV function can be detected by 4D flow MRI, but not by the conventional MRI and echocardiographic indices. The relative amount and energetics of RV inflow passing directly to outflow (Direct Flow) were lower in patients with higher degree of LV remodeling compared to patients with lower degree of LV remodeling and healthy subjects. These 4D flow-specific alterations suggest novel pathophysiological aspects of interventricular interaction that could add to the assessment of integrated cardiac function.

\section{Acknowledgements}

Thanks to Sven Petersson for technical assistance and Andreas Bussman and Johan Kihlberg for valuable input. 


\section{References}

1. Haddad F, Doyle R, Murphy DJ, Hunt Sa. Right ventricular function in cardiovascular disease, part II: pathophysiology, clinical importance, and management of right ventricular failure. Circulation 2008;117:1717-1731.

2. de Groote P, Millaire A, Foucher-Hossein C, et al. Right ventricular ejection fraction is an independent predictor of survival in patients with moderate heart failure. J Am Coll Cardiol 1998;32(4):948-954.

3. Di Salvo TG, Mathier M, Semigran MJ, Dec GW. Preserved right ventricular ejection fraction predicts exercise capacity and survival in advanced heart failure. J Am Coll Cardiol 1995;25(5):1143-1153.

4. Meluzin J, Spinarova L, Hude P, et al. Prognostic importance of various echocardiographic right ventricular functional parameters in patients with symptomatic heart failure. J Am Soc Echocardiogr 2005;18(5):435-444.

5. Larose E, Ganz P, Reynolds HG, et al. Right ventricular dysfunction assessed by cardiovascular magnetic resonance imaging predicts poor prognosis late after myocardial infarction. J Am Coll Cardiol 2007;49(8):855-862.

6. Antoni ML, Scherptong RW, Atary JZ, et al. Prognostic value of right ventricular function in patients after acute myocardial infarction treated with primary percutaneous coronary intervention. Circ Cardiovasc Imaging 2010;3(3):264-271.

7. Voelkel NF, Quaife Ra, Leinwand La, et al. Right ventricular function and failure: report of a National Heart, Lung, and Blood Institute working group on cellular and molecular mechanisms of right heart failure. Circulation 2006;114:1883-1891.

8. Rudski LG, Lai WW, Afilalo J, et al. Guidelines for the echocardiographic assessment of the right heart in adults: a report from the American Society of Echocardiography endorsed by the European Association of Echocardiography, a registered branch of the European Society of Cardiology, and t. Journal of the American Society of Echocardiography : official publication of the American Society of Echocardiography 2010;23:685-713; quiz 786-688.

9. Jurcut R, Giusca S, La Gerche A, Vasile S, Ginghina C, Voigt J-U. The echocardiographic assessment of the right ventricle: what to do in 2010? European journal of echocardiography : the journal of the Working Group on Echocardiography of the European Society of Cardiology 2010;11:81-96.

10. Lang RM, Badano LP, Mor-Avi V, et al. Recommendations for cardiac chamber quantification by echocardiography in adults: an update from the american society of echocardiography and the European association of cardiovascular imaging. J Am Soc Echocardiogr 2015;28(1):1-39 e14.

11. Richter Y, Edelman ER. Cardiology is flow. Circulation 2006;113:2679-2682.

12. Bolger AF, Heiberg E, Karlsson M, et al. Transit of blood flow through the human left ventricle mapped by cardiovascular magnetic resonance. Journal of cardiovascular magnetic resonance : official journal of the Society for Cardiovascular Magnetic Resonance 2007;9:741-747.

13. Kilner PJ, Yang GZ, Wilkes aJ, Mohiaddin RH, Firmin DN, Yacoub MH. Asymmetric redirection of flow through the heart. Nature 2000;404:759-761.

14. Carlsson M, Heiberg E, Toger J, Arheden H. Quantification of left and right ventricular kinetic energy using four-dimensional intracardiac magnetic 
resonance imaging flow measurements. Am J Physiol Heart Circ Physiol 2012;302(4):H893-900.

15. François CJ, Srinivasan S, Schiebler ML, et al. 4D cardiovascular magnetic resonance velocity mapping of alterations of right heart flow patterns and main pulmonary artery hemodynamics in tetralogy of Fallot. Journal of cardiovascular magnetic resonance : official journal of the Society for Cardiovascular Magnetic Resonance 2012;14:16.

16. Elbaz MS, Calkoen EE, Westenberg JJ, Lelieveldt BP, Roest AA, van der Geest RJ. Vortex flow during early and late left ventricular filling in normal subjects: quantitative characterization using retrospectively-gated 4D flow cardiovascular magnetic resonance and three-dimensional vortex core analysis. J Cardiovasc Magn Reson 2014;16(1):78.

17. Geiger J, Markl M, Jung B, et al. 4D-MR flow analysis in patients after repair for tetralogy of Fallot. European radiology 2011;21:1651-1657.

18. Fredriksson AG, Zajac J, Eriksson J, et al. 4-D blood flow in the human right ventricle. American journal of physiology Heart and circulatory physiology 2011;301:H2344-2350.

19. Guglin M, Verma S. Right side of heart failure. Heart Fail Rev 2012;17(3):511-527.

20. Buckberg GD. The ventricular septum: the lion of right ventricular function, and its impact on right ventricular restoration. European journal of cardiothoracic surgery : official journal of the European Association for Cardiothoracic Surgery 2006;29 Suppl 1:S272-278.

21. Maceira AM, Prasad SK, Khan M, Pennell DJ. Normalized left ventricular systolic and diastolic function by steady state free precession cardiovascular magnetic resonance. Journal of cardiovascular magnetic resonance : official journal of the Society for Cardiovascular Magnetic Resonance 2006;8:417426.

22. Eriksson J, Carlhäll CJ, Dyverfeldt P, Engvall J, Bolger AF, Ebbers T. Semiautomatic quantification of 4D left ventricular blood flow. Journal of cardiovascular magnetic resonance : official journal of the Society for Cardiovascular Magnetic Resonance 2010;12:9.

23. Heiberg E, Sjögren J, Ugander M, Carlsson M, Engblom H, Arheden H. Design and validation of Segment--freely available software for cardiovascular image analysis. BMC medical imaging 2010;10:1.

24. Wigström L, Ebbers T, Fyrenius a, et al. Particle trace visualization of intracardiac flow using time-resolved 3D phase contrast MRI. Magnetic resonance in medicine : official journal of the Society of Magnetic Resonance in Medicine / Society of Magnetic Resonance in Medicine 1999;41:793-799.

25. Nagueh SF, Appleton CP, Gillebert TC, et al. Recommendations for the evaluation of left ventricular diastolic function by echocardiography. J Am Soc Echocardiogr 2009;22(2):107-133.

26. Hill JA, Olson EN. Cardiac plasticity. N Engl J Med 2008;358(13):1370-1380.

27. Schwarz K, Singh S, Dawson D, Frenneaux MP. Right ventricular function in left ventricular disease: pathophysiology and implications. Heart Lung Circ 2013;22(7):507-511.

28. Saleh S, Liakopoulos OJ, Buckberg GD. The septal motor of biventricular function. Eur J Cardiothorac Surg 2006;29 Suppl 1:S126-138.

29. Watanabe H, Sugiura S, Hisada T. The looped heart does not save energy by maintaining the momentum of blood flowing in the ventricle. American 
journal of physiology Heart and circulatory physiology 2008;294:H21912196.

30. Pasipoularides A, Shu M, Shah A, Womack MS, Glower DD. Diastolic right ventricular filling vortex in normal and volume overload states. Am J Physiol Heart Circ Physiol 2003;284(4):H1064-1072. 


\section{Tables}

Table 1. Demographic and descriptive clinical data for the three groups given as mean $\pm \mathrm{SD}$.

\begin{tabular}{lccc}
\hline Variable & $\begin{array}{c}\text { Healthy controls } \\
(\mathbf{n}=\mathbf{1 1})\end{array}$ & $\begin{array}{c}\text { Lower-LVEDVI } \\
(\mathbf{n}=\mathbf{1 1})\end{array}$ & $\begin{array}{c}\text { Higher-LVEDVI } \\
(\mathbf{n}=\mathbf{1 1})\end{array}$ \\
\hline Age (years) & $67 \pm 4^{*}$ & $71 \pm 4$ & $69 \pm 5$ \\
Gender (M/F) & $2 / 9$ & $3 / 8$ & $171 \pm 8$ \\
Height (cm) & $169 \pm 8$ & $168 \pm 7$ & $75 \pm 13$ \\
Weight (kg) & $68 \pm 8$ & $78 \pm 15$ & $66 \pm 10$ \\
Heart rate (bpm) & $67 \pm 9$ & $66 \pm 10$ & $133 \pm 12$ \\
& $131 \pm 15^{*}$ & $146 \pm 11^{\dagger}$ & $71 \pm 11$ \\
SBP at rest (mmHg) & $78 \pm 7$ & $80 \pm 6^{\dagger}$ & \\
DBP at rest (mmHg) & & & \\
\hline
\end{tabular}

${ }^{*} \mathrm{P}<0.05$ vs. lower-LVEDVI. ${ }^{\dagger} \mathrm{P}<0.05$ vs. higher-LVEDVI

SBP, systolic blood pressure; DBP, diastolic blood pressure. 
Table 2. Descriptive MRI derived conventional parameters and LV E/é-ratio for assessment of ventricular function in the three groups given as mean \pm SD.

Variable

Healthy controls Lower-LVEDVI Higher-LVEDVI

\begin{tabular}{lccc} 
& $(\mathbf{n}=\mathbf{1 1})$ & $(\mathbf{n}=\mathbf{1 1})$ & $(\mathbf{n}=\mathbf{1 1})$ \\
\hline LVEDVI $\left(\mathrm{ml} / \mathrm{m}^{2}\right)$ & $67 \pm 12^{\dagger \dagger}$ & $62 \pm 10^{\dagger \dagger \dagger}$ & $98 \pm 32$ \\
RVEDVI $\left(\mathrm{ml} / \mathrm{m}^{2}\right)$ & $64 \pm 11$ & $59 \pm 8$ & $66 \pm 13$ \\
LV Ejection fraction (\%) & $64 \pm 7^{\dagger \dagger}$ & $68 \pm 7^{\dagger \dagger \dagger}$ & $48 \pm 13$ \\
RV Ejection fraction (\%) & $60 \pm 7$ & $58 \pm 10$ & $54 \pm 5$ \\
LV E/é ratio & $8.5 \pm 2.2^{\dagger}$ & $12.9 \pm 3.6$ & $12.2 \pm 4.1$
\end{tabular}

${ }^{*} \mathrm{P}<0.05$ vs. lower-LVEDVI. ${ }^{\dagger} \mathrm{P}<0.05,{ }^{\dagger \dagger} \mathrm{P}<0.01,{ }^{\dagger \dagger} \mathrm{P}<0.001$ vs. higher-LVEDVI.

$\mathrm{LV}$, left ventricle; RV, right ventricular; EDVI, end-diastolic volume index (EDV/body surface area); LV E/é-ratio, transmitral inflow Doppler /tissue Doppler velocity-ratio at early filling. 
Table 3. RV parameters from conventional echocardiographic examination for the three groups given as mean $\pm \mathrm{SD}$.

\begin{tabular}{lccc}
\hline Variable & Healthy controls & Lower-LVEDVI & Higher-LVEDVI \\
& $(\mathbf{n}=\mathbf{1 1})$ & $(\mathbf{n}=\mathbf{1 1})$ & $(\mathbf{n}=\mathbf{1 1})$ \\
\hline RV basal diameter $(\mathrm{mm})$ & $32 \pm 4$ & $34 \pm 6$ & $36 \pm 4$ \\
é/á ratio & $0.76 \pm 0.22$ & $0.61 \pm 0.18$ & $0.58 \pm 0.38$ \\
RA area $\left(\mathrm{cm}^{2}\right)$ & $14 \pm 3$ & $15 \pm 5$ & $16 \pm 3$ \\
RV FAC $(\%)$ & $44 \pm 7$ & $43 \pm 12$ & $45 \pm 7$ \\
TAPSE $(\mathrm{mm})$ & $23 \pm 4$ & $22 \pm 4$ & $22 \pm 4$ \\
RV s' $(\mathrm{cm} / \mathrm{s})$ & $11 \pm 2$ & $10 \pm 2$ & $9 \pm 3$ \\
& & & \\
\hline
\end{tabular}

$\mathrm{RV}$, right ventricle; é/á ratio, tissue peak velocity data in the RV free wall from early

(é) and late diastole (á), respectively; RA, right atrium; FAC, fractional area change;

TAPSE, tricuspid annular plane systolic excursion; s', tissue peak velocity data in the RV free wall at systole. 
Table 4. Right ventricular 4D flow specific parameters in the three groups given as means $\pm \mathrm{SD}$.

Variable

\section{Healthy controls Lower-LVEDVI Higher-LVEDVI}

$$
(\mathbf{n}=11) \quad(\mathbf{n}=11) \quad(\mathbf{n}=11)
$$

DF/EDV volume ratio (\%)

$44 \pm 6^{\dagger} \quad 44 \pm 6^{\dagger} \quad 38 \pm 5$

RI/EDV volume ratio (\%)

$19 \pm 3$

$17 \pm 4^{\dagger}$

$21 \pm 5$

DEF/EDV volume ratio (\%)

$13 \pm 3^{\dagger+*}$

$17 \pm 5$

$18 \pm 3$

ResV/EDV volume ratio (\%)

$24 \pm 7$

$21 \pm 4$

$24 \pm 5$

DF/EDV KE ratio at ED (\%)

$65 \pm 7^{\dagger+\dagger}$

$64 \pm 7^{\dagger \dagger \dagger}$

$52 \pm 6$

$* \mathrm{P}<0.05$ vs. lower-LVEDVI. ${ }^{\dagger} \mathrm{P}<0.05,{ }^{\dagger \dagger} \mathrm{P}<0.01,{ }^{\dagger \dagger} \mathrm{P}<0.001$ vs. higher-LVEDVI.

DF, Direct flow; RI, Retained Inflow; DEF, Delayed Ejection Flow; ResV, Residual

Volume; EDV, end-diastolic volume; KE, kinetic energy; ED, end diastole. 


\section{Figure legends}

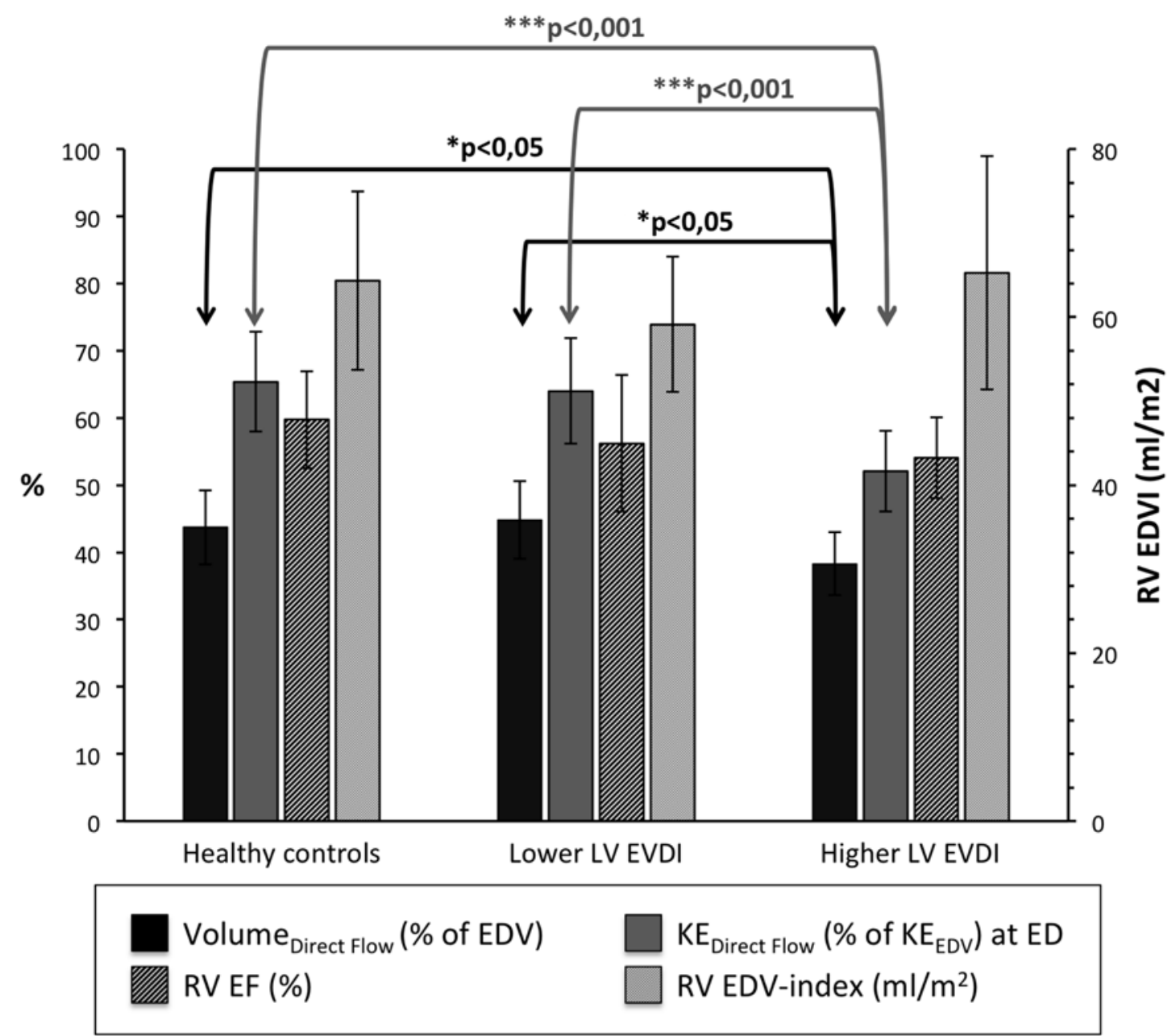

Figure 1 - Graphic representation of 4D flow-specific and MRI derived conventional right ventricular (RV) parameters. The RV Direct Flow/EDV volume-ratio was significantly lower in the higher-LVEDVI group than in the other groups, and the kinetic energy (KE) possessed by the Direct Flow relative the KE possessed by the EDV at ED was also significantly lower in the higher-LVEDVI group than in the other groups. No significant difference was seen in the RVEF or RVEDVI between the groups. 


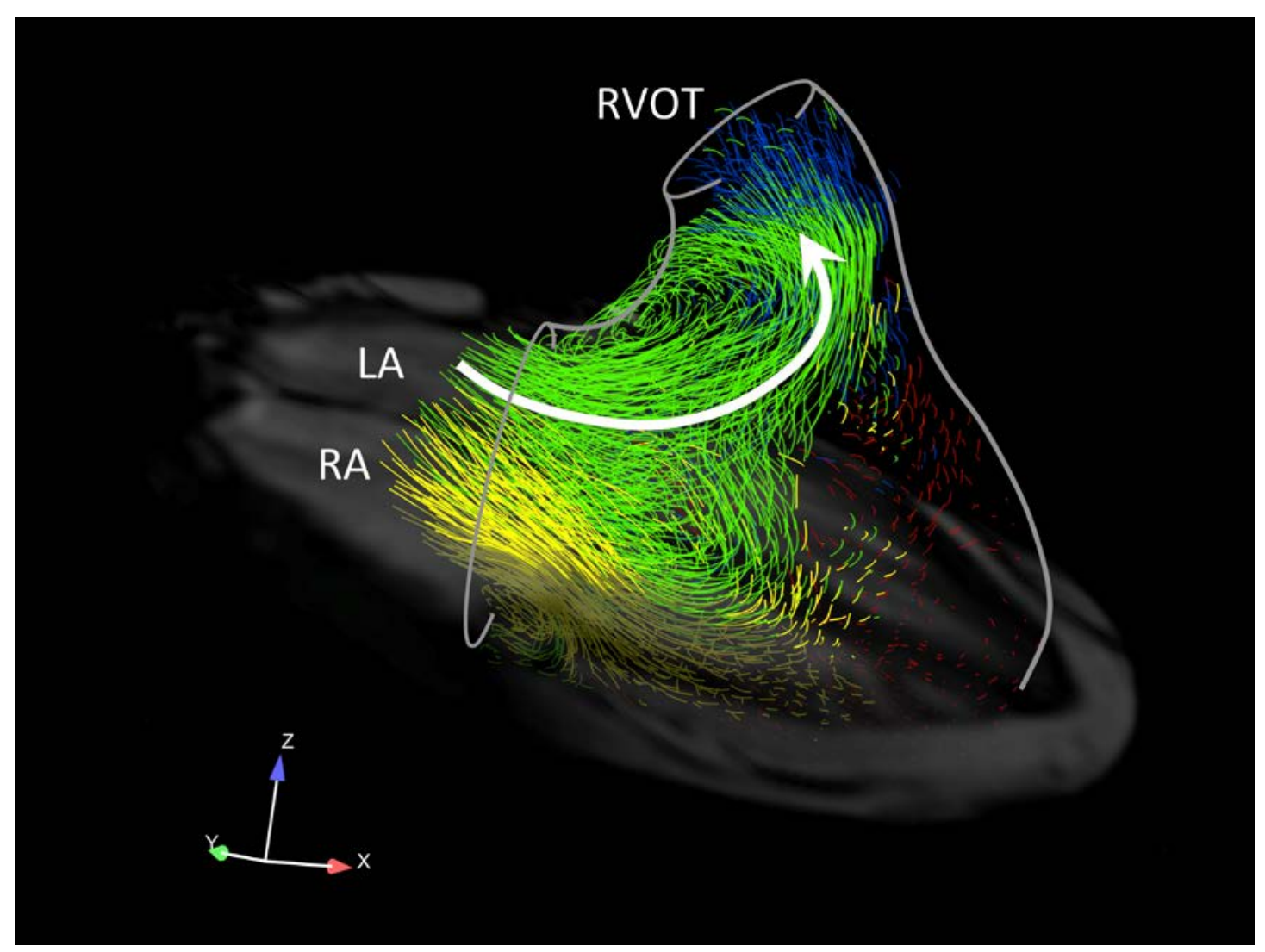

Figure 2 - Pathline visualization of right ventricular (RV) flow components at late diastolic filling in a 70 y.o. healthy subject with a LVEDVI of $61 \mathrm{ml} / \mathrm{m}^{2}$. The inflowing blood is directed towards the outflow tract through the ring vortex (white arrow). Direct flow, green; Retained inflow, yellow, Delayed ejection flow, blue; and Residual volume, red. Some pathlines in the lateral part of the RV is cut in order to visualize pathlines closer to the interventricular septum. A semi-transparent fourchamber image provides anatomical orientation. LA, left atrium; RA, right atrium; RVOT, right ventricular outflow tract. 


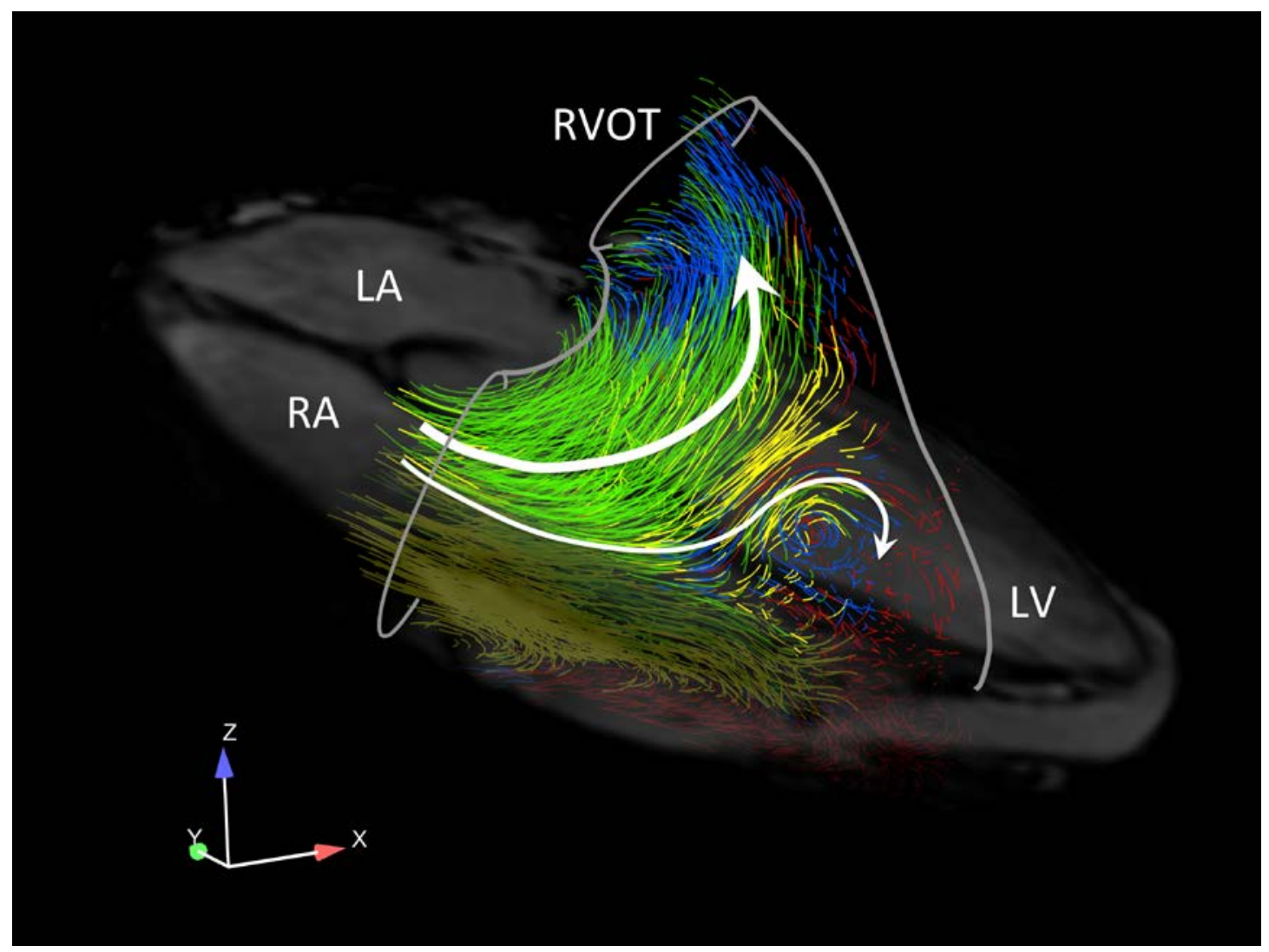

Figure 3 - Pathline visualization of right ventricular (RV) flow components at late diastolic filling in a 72 y.o. patient with a LVEDVI of $128 \mathrm{ml} / \mathrm{m}^{2}$. The inflowing blood in the superior portion of the ring vortex is divided into a major counter clockwise flow path up into the RVOT (large white arrow) (mainly Direct flow) but also into a minor clockwise flow path down towards the apical part of the interventricular septum (small white arrow). Direct flow, green; Retained inflow, yellow, Delayed ejection flow, blue; and Residual volume, red. Some pathlines in the lateral part of the RV is cut in order to visualize pathlines closer to the interventricular septum. A semi-transparent four-chamber image provides anatomical orientation. LA, left atrium; RA, right atrium; RVOT, right ventricular outflow tract. 


\section{Movies}

Supplemental movie 1 - Pathline visualization of right ventricular (RV) flow components throughout a cardiac cycle in a 70 y.o. healthy subject with a LVEDVI of $61 \mathrm{ml} / \mathrm{m}^{2}$. Direct flow, green; Retained inflow, yellow; Delayed ejection flow, blue; and Residual volume, red. A semi-transparent four-chamber image provides anatomical orientation.

Supplemental movie 2 - Pathline visualization of right ventricular (RV) flow components throughout a cardiac cycle in a 72 y.o. patient with a LVEDVI of 128 $\mathrm{ml} / \mathrm{m}^{2}$. Direct flow, green; Retained inflow, yellow; Delayed ejection flow, blue; and Residual volume, red. A semi-transparent four-chamber image provides anatomical orientation. 